\title{
Mappings with a Common Fixed Point in Generalized $D^{*}$-Metric Spaces
}

\author{
Sandeep Bhatt, Brian Fisher and Shruti Chaukiyal
}

\begin{abstract}
The purpose of this paper is to establish a common fixed point theorem in a generalized $D^{*}$-metric space. Our results unify, generalize and complement the comparable results from the current literature.
\end{abstract}

\section{Introduction AND Preliminaries}

Dhage [2] introduced the notion of generalized metric spaces (D-metric spaces) in 1992. He proved the existence of a unique fixed point of a self-map satisfying a contractive condition in complete and bounded D-metric spaces. In a subsequent series of papers Dhage attempted to develop topological structures in such spaces (see, for instance [3], [4], and [5]). He claimed that $D$-metric provide a generalization of ordinary metric functions and went on to present several fixed point results. In 2004, Mustafa and Sims [9] demonstrated that the claims concerning the fundamental topological structure of $D$-metric spaces are incorrect and introduced more appropriate notion of $D$-metric spaces. In 2007, Sedghi, Shobe and Zhoh [7] introduced the notion of $D^{*}$-metric spaces which is a modification of the definition of $D$-metric spaces and proved a common fixed point theorem for a class of mappings in complete $D^{*}$-metric spaces.

Huang and Zhang [6] introduced the concept of a cone metric space, replacing the set of real numbers by an ordered Banach space and obtained some fixed point theorems for mappings satisfying different contractive conditions.

Let $E$ be a real Banach space and $P$ a subset of $E$. The set $P$ is called a cone if and only if

(i) $P$ is closed, non-empty and $P \neq\{0\}$;

(ii) $a, b \in R, a, b \geq 0, x, y \in P$ imply that $a x+b y \in P$;

(iii) $P \cap(-P)=\{0\}$.

For a given cone $P \subset E$ we define a partial ordering $\leq$ with respect to $P$ by $x \leq y$ if and only if $y-x \in P$. We write $x<y$ to indicate that $x \rightarrow y$ but

2000 Mathematics Subject Classification. Primary: 47H10, 54H25.

Key words and phrases. Generalized $D^{*}$-metric space, normal cones, fixed point. 
$x \neq y$, while $x<<y$ will stand for $y-x \in \operatorname{int} P$ the interior of the set $P$. Let $E$ be a Banach space and $P \subset E$ a cone. The cone $P$ is called normal if there is a number $K>0$ such that

$$
0 \leq x \leq y \text { implies }\|x\| \leq K\|y\| \text { for all } \quad x, y \in E .
$$

The least positive number $K$ satisfying the above inequality is called the normal constant of $P$. In the following we always suppose that $E$ is a Banach space, $P$ is a cone in $E$ with int $P \neq \phi$ and $\leq$ is partial ordering with respect to $P$. Recently, Aage and Salunke introduced the notion of a generalized $D^{*}$-metric space by replacing $R$ by a real Banach space in $D^{*}$ metric space for all $x, y, z, w$ in $X$ and proved some fixed point theorems in complete generalized $D^{*}$ - metric spaces.

The following definitions and some basic results in generalized $D^{*}$-metric spaces are due to [1].

Definition 1.1. Let $X$ be a nonempty set. A generalized metric (or $D^{*}$ metric) on $X$ is a function $D^{*}: X^{3} \rightarrow E$ that satisfies the following conditions for each $x, y, z, w \in X$ :

(1) $D^{*}(x, y, z) \geq 0$

(2) $D^{*}(x, y, z)=0$ if and only if $x=y=z$,

(3) $D^{*}(x, y, z)=D^{*}(p\{x, y, z\})$ (symmetry) where $p$ is a permutation function,

(4) $D^{*}(x, y, z) \leq D^{*}(x, y, w)+D^{*}(w, z, z)$.

The pair $\left(X, D^{*}\right)$ is called a generalized metric (or $D^{*}$-metric) space.

Proposition 1.1. If $\left(X, D^{*}\right)$ be a generalized $D^{*}$-metric space, for all $x, y \in$ $X$, then we have $D^{*}(x, x, y)=D^{*}(x, y, y)$.

Definition 1.2. Let $\left(X, D^{*}\right)$ be a generalized $D^{*}$-metric space. Let $\left\{x_{n}\right\}$ be a sequence with $x$ a point in $X$. If for every $c \in E$ with $0<<c$ there is $N$ such that for all $m, n>N, D^{*}\left(x_{m}, x_{n}, x\right)<<c$, then $\left\{x_{n}\right\}$ is said to be convergent and $\left\{x_{n}\right\}$ converges to $x$ and $x$ is the limit of $\left\{x_{n}\right\}$. We denote this by $x_{n} \rightarrow x$ as $n \rightarrow \infty$.

Definition 1.3. Let $\left(X, D^{*}\right)$ be a generalized $D^{*}$-metric space, $P$ be a normal cone with normal constant $K$. Let $\left\{x_{n}\right\}$ be a sequence in $X$. Then $\left\{x_{n}\right\}$ converges to $x$ if and only if $D^{*}\left(x_{m}, x_{n}, x\right) \rightarrow 0$ as $m, n \rightarrow \infty$.

Lemma 1.1. Let $\left(X, D^{*}\right)$ be generalized $D^{*}$-metric space, then the following are equivalent:

(i) $\left\{x_{n}\right\}$ is $D^{*}$-convergent to $x$;

(ii) $D^{*}\left(x_{n}, x_{n}, x\right) \rightarrow 0$ (as $\left.n \rightarrow \infty\right)$;

(iii) $D^{*}\left(x_{n}, x, x\right) \rightarrow 0$ (as $\left.n \rightarrow \infty\right)$. 
Lemma 1.2. Let $\left(X, D^{*}\right)$ be a generalized $D^{*}$-metric space, $P$ be a normal cone with normal constant $K$. Let $\left\{x_{n}\right\}$ be a sequence in $X$. If $\left\{x_{n}\right\}$ converges to $x$ and $\left\{x_{n}\right\}$ converges to $y$, then $x=y$. That is the limit of $\left\{x_{n}\right\}$, if exists, is unique.

Definition 1.4. Let $\left(X, D^{*}\right)$ be a generalized $D^{*}$-metric space, $\left\{x_{n}\right\}$ be a sequence in $X$. If for any $c \in E$ with $0<<c$, there is $N$ such that for all $m, n, l>N, D^{*}\left(x_{m}, x_{n}, x_{l}\right)<<c$, then $\left\{x_{n}\right\}$ is called a Cauchy sequence in $X$.

Definition 1.5. Let $\left(X, D^{*}\right)$ be a generalized $D^{*}$-metric space. If every Cauchy sequence in $X$ is convergent in $X$, then $X$ is called a complete generalized $D^{*}$-metric space.

Lemma 1.3. Let $\left(X, D^{*}\right)$ be generalized $D^{*}$-metric space, $\left\{x_{n}\right\}$ be a sequence in $X$. if $\left\{x_{n}\right\}$ converges to $x$, then $\left\{x_{n}\right\}$ is a Cauchy sequence.

Lemma 1.4. Let $\left(X, D^{*}\right)$ be a generalized $D^{*}$-metric space, $P$ be a normal cone with normal constant $K$. Let $\left\{x_{n}\right\}$ be a sequence in $X$. Then $\left\{x_{n}\right\}$ is a Cauchy sequence if and only if $D^{*}\left(x_{m}, x_{n}, x_{l}\right) \rightarrow 0$ as $m, n, l \rightarrow \infty$.

Lemma 1.5. Let $\left(X, D^{*}\right)$ be a generalized $D^{*}$-metric space, $P$ be a normal cone with normal constant $K$. Let $\left\{x_{n}\right\},\left\{y_{n}\right\}$ and $\left\{z_{n}\right\}$ be three sequences in $X$ and let $x_{n} \rightarrow x, y_{n} \rightarrow y$ and $z_{n} \rightarrow z$ as $n \rightarrow \infty$. Then $D^{*}\left(x_{n}, y_{n}, z_{n}\right) \rightarrow$ $D^{*}(x, y, z)$ as $n \rightarrow \infty$.

\section{Main Results}

Theorem 2.1. Let $\left(X, D^{*}\right)$ be a generalized $D^{*}$-metric space, $P$ be a normal cone with normal constant $K$ and let $S, T: X \rightarrow X$, be two mappings which satisfies the following conditions:

(i) $T(X) \subset S(X)$,

(ii) $T(X)$ or $S(X)$ is $D^{*}$-complete, and

(iii) inequality:

$$
\begin{aligned}
D^{*}(T x, T y, T z) \leq & h \max \left\{D^{*}(S x, S y, S z),\right. \\
& D^{*}(S x, T x, T x), D^{*}(S y, T y, T y), D^{*}(S x, T y, T z), \\
& \left.D^{*}(S y, T z, T x), D^{*}(S z, T z, T z), D^{*}(S z, T x, T y)\right\}
\end{aligned}
$$

for all $x, y, z \in X$, where $0 \leq h<1$.

Then $S$ and $T$ have a unique coincident point in $X$. Moreover if $S$ and $T$ are weakly compatible, then $S$ and $T$ have a unique common fixed point.

Proof. Let $x_{0} \in X$ be a arbitrary, there exists $x_{1} \in X$ such that $T x_{0}=S x_{1}$, in this way we have a sequence $\left\{S x_{n}\right\}$ with $T x_{n-1}=S x_{n}$. Then from the 
inequality (2.1), we have

$$
\begin{aligned}
& D^{*}\left(T x_{n-1}, T x_{n}, T x_{n}\right) \leq h \max \left\{D^{*}\left(S x_{n-1}, S x_{n}, S x_{n}\right),\right. \\
& D^{*}\left(S x_{n-1}, T x_{n-1}, T x_{n-1}\right), D^{*}\left(S x_{n}, T x_{n}, T x_{n}\right), \\
& D^{*}\left(S x_{n}, T x_{n}, T x_{n}\right), D^{*}\left(S x_{n-1}, T x_{n}, T x_{n}\right), \\
&\left.D^{*}\left(S x_{n}, T x_{n-1}, T x_{n}\right), D^{*}\left(S x_{n}, T x_{n-1}, T x_{n}\right)\right\} \\
& \leq h \max \left\{D^{*}\left(S x_{n-1}, S x_{n}, S x_{n}\right), D^{*}\left(S x_{n-1}, S x_{n}, S x_{n}\right),\right. \\
& D^{*}\left(S x_{n}, S x_{n+1}, S x_{n+1}\right), D^{*}\left(S x_{n}, S x_{n+1}, S x_{n+1}\right), \\
& D^{*}\left(S x_{n-1}, S x_{n+1}, S x_{n+1}\right), D^{*}\left(S x_{n}, S x_{n}, S x_{n+1}\right), \\
&\left.D^{*}\left(S x_{n}, S x_{n}, S x_{n+1}\right)\right\} \\
& \leq h D^{*}\left(S x_{n-1}, S x_{n}, S x_{n}\right),
\end{aligned}
$$

where $0 \leq h<1$. By repeated application of above inequality we have

$$
D^{*}\left(S x_{n}, S x_{n+1}, S x_{n+1}\right) \leq h^{n} D^{*}\left(S x_{0}, S x_{1}, S x_{1}\right) .
$$

Then, for all $n, m \in N, n<m$ we have by repeated use of rectangle inequality and equality (2.2) that

$$
\begin{aligned}
D^{*}( & \left.S x_{n}, S x_{m}, S x_{m}\right) \leq D^{*}\left(S x_{n}, S x_{n}, S x_{n+1}\right)+D^{*}\left(S x_{n+1}, S x_{n+1}, S x_{n+2}\right)+ \\
& D^{*}\left(S x_{n+2}, S x_{n+2}, S x_{n+3}\right)+\cdots+D^{*}\left(S x_{m-1}, S x_{m-1}, S x_{m}\right) \\
\leq & D^{*}\left(S x_{n}, S x_{n+1}, S x_{n+1}\right)+D^{*}\left(S x_{n+1}, S x_{n+2}, S x_{n+2}\right)+\cdots+ \\
& D^{*}\left(S x_{m-1}, S x_{m}, S x_{m}\right) \\
\leq & \left(h^{n}+h^{n+1}+\cdots+h^{m-1}\right) D^{*}\left(S x_{0}, S x_{1}, S x_{1}\right) \\
\leq & \frac{h^{n}}{1-h} D^{*}\left(S x_{0}, S x_{1}, S x_{1}\right)
\end{aligned}
$$

and so

$$
\left\|D^{*}\left(S x_{n}, S x_{m}, S x_{m}\right)\right\| \leq \frac{h^{n}}{1-h} K\left\|D^{*}\left(S x_{0}, S x_{1}, S x_{1}\right)\right\| .
$$

This implies that $D^{*}\left(S x_{n}, S x_{m}, S x_{m}\right) \rightarrow 0$, as $n, m \rightarrow \infty$, since

$$
\begin{gathered}
\frac{h^{n}}{1-h} K\left\|D^{*}\left(S x_{0}, S x_{1}, S x_{1}\right)\right\| \rightarrow 0, \quad \text { as } \quad n, m \rightarrow \infty, \quad \text { for } \quad n, m, l \in N, \\
D^{*}\left(S x_{n}, S x_{m}, S x_{l}\right) \leq D^{*}\left(S x_{n}, S x_{m}, S x_{m}\right)+D^{*}\left(S x_{m}, S x_{l}, S x_{l}\right),
\end{gathered}
$$

from (1.1), we have,

$$
\left\|D^{*}\left(S x_{n}, S x_{m}, S x_{l}\right)\right\| \leq K\left[\left\|D^{*}\left(S x_{n}, S x_{m}, S x_{m}\right)\right\|+\left\|D^{*}\left(S x_{m}, S x_{l}, S x_{l}\right)\right\|\right] .
$$

Taking the limit as $n, m, l \rightarrow \infty$, we get $D^{*}\left(S x_{n}, S x_{m}, S x_{l}\right) \rightarrow 0$. So $\left\{S x_{n}\right\}=\left\{T x_{n-1}\right\}$ is a $D^{*}$-Cauchy sequence. Since $S(X)$ is a $D^{*}$-complete, there exists $u \in S(X)$ such that $\left\{S x_{n}\right\} \rightarrow u$ as $n \rightarrow \infty$. Then there exists $p \in X$ such that $S p=u$. If $T(x)$ is $D^{*}$-complete, there exists $u \in T(X)$ 
such that $\left\{T x_{n-1}\right\} \rightarrow u$ and since $T(X) \subset S(X)$, we have $u \in S(X)$. Then there exists $p \in X$ such that $S p=u$.

We claim that $T p=u$,

$$
\begin{aligned}
& D^{*}(T p, u, u) \leq D^{*}\left(T p, T p, T x_{n}\right)+D^{*}\left(T x_{n}, u, u\right) \\
& \leq h \max \left\{D^{*}\left(S p, S p, S x_{n}\right), D^{*}(S p, T p, T p), D^{*}(S p, T p, T p),\right. \\
& D^{*}\left(S x_{n}, T x_{n}, T x_{n}\right), D^{*}\left(S p, T x_{n}, T p\right), D^{*}\left(S p, T p, T x_{n}\right), \\
& \left.D^{*}\left(S x_{n}, T p, T p\right)\right\}+D^{*}\left(T x_{n}, u, u\right) \\
& \leq h \max \left\{D^{*}\left(u, u, S x_{n}\right), D^{*}(u, T p, T p), D^{*}(u, T p, T p),\right. \\
& D^{*}\left(S x_{n}, S x_{n+1}, S x_{n+1}\right), D^{*}\left(u, S x_{n+1}, T p\right), D^{*}\left(u, T p, S x_{n+1}\right), \\
& \left.D^{*}\left(S x_{n}, T p, T p\right)\right\}+D^{*}\left(T x_{n}, u, u\right) \\
& D^{*}(T p, u, u) \leq h \max \left\{D^{*}\left(u, u, S x_{n}\right), D^{*}(u, T p, T p),\right. \\
& \quad D^{*}\left(S x_{n}, S x_{n+1}, S x_{n+1}\right), D^{*}\left(u, S x_{n+1}, T p\right), \\
& \left.\quad D^{*}\left(S x_{n}, T p, T p\right)\right\}+D^{*}\left(S x_{n+1}, u, u\right)
\end{aligned}
$$

and so,

$$
\begin{aligned}
\left\|D^{*}(T p, T p, u)\right\| \leq & K h \max \left\{\left\|D^{*}\left(u, u, S x_{n}\right)\right\|,\left\|D^{*}(u, T p, T p)\right\|,\right. \\
& \left\|D^{*}\left(S x_{n}, S x_{n+1}, S x_{n+1}\right)\right\|,\left\|D^{*}\left(u, S x_{n+1}, T p\right)\right\|, \\
& \left.\left\|D^{*}\left(S x_{n}, u, T p\right)\right\|\right\}+\left\|D^{*}\left(S x_{n+1}, u, u\right)\right\| .
\end{aligned}
$$

As $n \rightarrow \infty$, the right hand side tends to zero. Hence $\left\|D^{*}(T p, T p, u)\right\|=0$ and $T p=u$, i.e., $T p=S p$ and $p$ is a coincident point of $S$ and $T$. Now we show that $S$ and $T$ have a unique coincident point. For this, assume that there exists a point $q$ in $X$ such that $S q=T q$. Now,

$$
\begin{aligned}
D^{*}( & T p, T p, T q) \leq h \max \left\{D^{*}(S p, S p, S q), D^{*}(S p, T p, T p), D^{*}(S p, S p, T p),\right. \\
& \left.D^{*}(S q, T q, T q), D^{*}(S p, T p, T q), D^{*}(S p, T q, T p), D^{*}(S q, T p, T p)\right\} \\
\leq & h \max \left\{D^{*}(S p, S p, S q), 0,0,0, D^{*}(S p, T q, T p), D^{*}(S q, T p, T p)\right\} \\
\leq & h \max \left\{D^{*}(T p, T p, T q), 0,0,0, D^{*}(T p, T q, T p), D^{*}(T q, T p, T p)\right\} \\
= & h D^{*}(T p, T p, T q),
\end{aligned}
$$

and so we have $D^{*}(T p, T p, T q) \leq h D^{*}(T p, T p, T q)$, i.e., $(h-1) D^{*}(T p, T p, T q)$ $\in P$. However, $(h-1) D^{*}(T p, T p, T q) \in-P$, since $h-1<0$ and hence $(h-1) D^{*}(T p, T p, T q)=0$. This implies that $D^{*}(T p, T p, T q)=0$, i.e., $T p=T q$. Thus $p$ is the unique coincident point of $S$ and $T$. So $S$ and $T$ have a unique common fixed point. 
Corollary 2.1. Let $\left(X, D^{*}\right)$ be a generalized $D^{*}$-metric space, $P$ be a normal cone with normal constant $K$ and let $T: X \rightarrow X$, be a mapping which satisfies the following conditions:

$$
\begin{gathered}
D^{*}(T x, T y, T z) \leq h \max \left\{D^{*}(x, y, z), D^{*}(x, T x, T x), D^{*}(y, T y, T y),\right. \\
\left.D^{*}(x, T y, T y), D^{*}(y, T x, T x), D^{*}(z, T z, T z), D^{*}(z, T y, T y)\right\}
\end{gathered}
$$

for all $x, y, z \in X$, where $0 \leq h<1$. Then $T$ has a unique fixed point in $X$.

Theorem 2.2. Let $\left(X, D^{*}\right)$ be a generalized $D^{*}$-metric space, $P$ be a normal cone with normal constant $K$ and let $S, T: X \rightarrow X$, be two mappings which satisfies the following conditions

(i) $T(X) \subset S(X)$,

(ii) $T(X)$ or $S(X)$ is $D^{*}$-complete, and

(iii) inequality

$$
\begin{gathered}
D^{*}(T x, T y, T z) \leq h \max \left\{D^{*}(S x, S y, S z), D^{*}(S x, T x, T x),\right. \\
\left.D^{*}(S y, T y, T y)\right\}
\end{gathered}
$$

for all $x, y, z \in X$, where $0 \leq h<\frac{1}{2}$.

Then $S$ and $T$ have a unique coincident point in $X$.

Proof. Let $x_{0} \in X$ be arbitrary, there exists $x_{1} \in X$ such that $T x_{0}=S x_{1}$, in this way we have a sequence $\left\{S x_{n}\right\}$ with $T x_{n-1}=S x_{n}$. Then from the inequality (2.3), we have

$$
\begin{aligned}
D^{*}( & \left.S x_{n}, S x_{n+1}, S x_{n+1}\right)=D^{*}\left(T x_{n-1}, T x_{n}, T x_{n}\right) \\
\leq & h \max \left\{D^{*}\left(S x_{n-1}, S x_{n}, S x_{n}\right), D^{*}\left(S x_{n-1}, T x_{n-1}, T x_{n-1}\right),\right. \\
& \left.D^{*}\left(S x_{n}, T x_{n}, T x_{n}\right)\right\} \\
\leq & h \max \left\{D^{*}\left(S x_{n-1}, S x_{n}, S x_{n}\right), D^{*}\left(S x_{n-1}, S x_{n}, S x_{n}\right),\right. \\
& \left.D^{*}\left(S x_{n}, S x_{n+1}, S x_{n+1}\right)\right\} \\
\leq & h D^{*}\left(S x_{n-1}, S x_{n}, S x_{n}\right) .
\end{aligned}
$$

This implies that

$$
D^{*}\left(S x_{n}, S x_{n+1}, S x_{n+1}\right) \leq h D^{*}\left(S x_{n-1}, S x_{n}, S x_{n}\right)
$$

where $0 \leq h<\frac{1}{2}$. By repeated application of above inequality we have

$$
D^{*}\left(S x_{n}, S x_{n+1}, S x_{n+1}\right) \leq h^{n} D^{*}\left(S x_{0}, S x_{1}, S x_{1}\right) .
$$


Then, for all $n, m \in N, n<m$ we have by repeated use of rectangle inequality

$$
\begin{aligned}
D^{*} & \left.S x_{n}, S x_{m}, S x_{m}\right) \leq D^{*}\left(S x_{n}, S x_{n}, S x_{n+1}\right)+D^{*}\left(S x_{n+1}, S x_{n+1}, S x_{n+2}\right)+ \\
& D^{*}\left(S x_{n+2}, S x_{n+2}, S x_{n+3}\right)+\cdots+D^{*}\left(S x_{m-1}, S x_{m-1}, S x_{m}\right) \\
\leq & D^{*}\left(S x_{n}, S x_{n+1}, S x_{n+1}\right)+D^{*}\left(S x_{n+1}, S x_{n+2}, S x_{n+2}\right)+\cdots+ \\
& D^{*}\left(S x_{m-1}, S x_{m}, S x_{m}\right) \\
\leq & \left(h^{n}+h^{n+1}+\cdots+h^{m-1}\right) D^{*}\left(S x_{0}, S x_{1}, S x_{1}\right) .
\end{aligned}
$$

From (1.1), we have

$$
D^{*}\left(S x_{n}, S x_{m}, S x_{m}\right) \leq \frac{h^{n}}{1-h} D^{*}\left(S x_{0}, S x_{1}, S x_{1}\right)
$$

and so,

$$
\left\|D^{*}\left(S x_{n}, S x_{m}, S x_{m}\right)\right\| \leq \frac{h^{n}}{1-h} K\left\|D^{*}\left(S x_{0}, S x_{1}, S x_{1}\right)\right\|,
$$

which implies that $D^{*}\left(S x_{n}, S x_{m}, S x_{m}\right) \rightarrow 0$, as $n, m \rightarrow \infty$, since

$$
\frac{h^{n}}{1-h} K\left\|D^{*}\left(S x_{0}, S x_{1}, S x_{1}\right)\right\| \rightarrow 0
$$

as $n, m \rightarrow \infty$.

Since $0 \leq h<\frac{1}{2},\left\{S x_{n}\right\}$ is $D^{*}$-Cauchy sequence. By the completeness of $S(X)$, there exists $u \in S(X)$ such that $\left\{S x_{n}\right\}$ is $D^{*}$-convergent to $u$. Then there is $p \in X$, such that $S p=u$. If $T(X)$ is complete, then there exist $u \in T(X)$ such that $S x_{n} \rightarrow u$, as $T(X) \subset S(X)$, we have $u \in S(X)$. Then there exist $p \in X$ such that $S p=u$.

We claim that $T p=u$.

$$
\begin{aligned}
& D^{*}(T p, u, u)=D^{*}(T p, T p, u) \\
& \leq D^{*}\left(T p, T p, T x_{n}\right)+D^{*}\left(T x_{n}, u, u\right) \\
& \leq h \max \left\{D^{*}\left(S p, S p, S x_{n}\right), D^{*}(S p, T p, T p),\right. \\
& \left.\quad D^{*}(S p, T p, T p)\right\}+D^{*}\left(T x_{n}, u, u\right) \\
& \leq h \max \left\{D^{*}(S p, T p, T p), D^{*}\left(S p, S p, S x_{n}\right)\right\}+D^{*}\left(T x_{n}, u, u\right) \\
& \left\|D^{*}(T p, T p, u)\right\| \leq K h \max \left\{\left\|D^{*}(S p, T p, T p)\right\|,\left\|D^{*}\left(S p, S p, S x_{n}\right)\right\|\right\} \\
& +\left\|D^{*}\left(S x_{n+1}, u, u\right)\right\| .
\end{aligned}
$$

Hence,

$$
\left\|D^{*}(T p, T p, u)\right\| \leq K h \max \left\{\left\|D^{*}(u, T p, T p)\right\|, 0\right\}+\left\|D^{*}(u, u, u)\right\| .
$$

The right hand side tends to zero as $n \rightarrow \infty$. Hence $\left\|D^{*}(T p, T p, u)\right\|=0$ and $T p=u$. Hence $T p=S p$ and $p$ is a coincident point of $S$ and $T$. 
Now we show that $S$ and $T$ have a unique coincident point. For this, assume that there exists a point $q$ in $X$ such that $S q=T q$. Now

$$
\begin{aligned}
D^{*}(T p, T p, T q) & \leq h \max \left\{D^{*}(S p, S p, S q), D^{*}(S p, T p, T p), D^{*}(S p, S p, T p)\right. \\
& \leq h \max \left\{0,0, D^{*}(T p, T p, T q)\right\}
\end{aligned}
$$

This implies $(h-1) D^{*}(T p, T p, T q) \in P$ and $(h-1) D^{*}(T p, T p, T q) \in-P$ since $0 \leq h<\frac{1}{2}$. As $P \cap-P=\{0\}$, we have $(h-1) D^{*}(T p, T p, T q)=0$, i.e., $D^{*}(T p, T p, T q)=0$. Hence $T p=T q$. Also $S p=S q$, since $T p=S p$. Hence $p$ is the unique coincident point of $S$ and $T$. So $p$ is a unique common fixed point of $S$ and $T$ in $X$.

Corollary 2.2. Let $\left(X, D^{*}\right)$ be a generalized $D^{*}$-metric space, $P$ be a normal cone with normal constant $K$ and let $T: X \rightarrow X$, be a mapping which satisfies the following conditions

$$
D^{*}(T x, T y, T z) \leq h \max \left\{D^{*}(x, y, z), D^{*}(x, T x, T x), D^{*}(y, T y, T y)\right\}
$$

for all $x, y, z \in X$, where $0 \leq h<1$. Then $T$ has a unique fixed point in $X$.

Example 2.1. Let $\left(X, D^{*}\right)$ be a complete $D^{*}$-metric space, where $X=(0,1]$ and $D^{*}(x, y, z)=|x-y|+|y-z|+|z-x|$. Define self-maps $S$ and $T$ on $X$ as follows: $S x=\frac{x+1}{2}$ and $T x=\frac{x+5}{6}$, for all $x \in X$. For any nonzero $x \in X$ we have

$$
S T x=S\left(\frac{x+5}{6}\right)=\frac{x+11}{6}, \quad T S x=T\left(\frac{x+1}{2}\right)=\frac{x+11}{6} .
$$

Since $S T x=T S x$ and $S, T$ are weakly compatible on $X$.

Now

$$
\begin{aligned}
D^{*}(\text { ST } x, \text { TS } x, T S x)= & \left|\frac{x+11}{12}-\frac{x+11}{12}\right|+\left|\frac{x+11}{12}-\frac{x+11}{12}\right|+ \\
& +\left|\frac{x+11}{12}-\frac{x+11}{12}\right|=0, \\
D^{*}(S x, T x, T x)= & \left|\frac{x+1}{2}-\frac{x+5}{6}\right|+\left|\frac{x+5}{6}-\frac{x+5}{6}\right|+ \\
& +\left|\frac{x+1}{2}-\frac{x+5}{6}\right|=\frac{2 x-2}{3} .
\end{aligned}
$$

We see that

$$
D^{*}(S T x, T S x, T S x) \leq D^{*}(S x, T x, T x),
$$


and so $\{A, S\}$ are weakly commuting pairs.

$$
\begin{aligned}
& D^{*}(T x, T y, T z)=D^{*}\left(\frac{x+5}{6}, \frac{y+5}{6}, \frac{z+5}{6}\right) \\
&=\left|\frac{x+5}{6}-\frac{y+5}{6}\right|+\left|\frac{y+5}{6}-\frac{z+5}{6}\right|+\left|\frac{x+5}{6}-\frac{z+5}{6}\right| \\
&=\frac{(x-y-z)}{3} \\
& h \max \left\{D^{*}(S x, S y, S z), D^{*}(S x, T x, T x), D^{*}(S y, T y, T y)\right\}= \\
& h \max \left\{(x-y-z), \frac{2 x-2}{3}, \frac{2 y-2}{3}\right\}
\end{aligned}
$$

for all $x, y, z \in X, h \in\left(0, \frac{1}{2}\right]$, Theorem 2.2 is satisfied. So 1 is the unique common fixed point for $S$ and $T$.

\section{REFERENCES}

[1] C. T. Aage and J. N. Salunke, Some fixed points theorems in generalized $D^{*}$-metric spaces, Applied Sciences, vol.12 (2010), 1-13.

[2] B.C. Dhage, Generalized metric spaces and mappings with fixed point, Bull. Cal. Math. Soc. 84(1992), 329-336.

[3] B.C. Dhage, Generalized metric spaces and topological Structure. I, Analele Stiintifice ale Universitatii Al. I. Cuza din Iasi.Serie Noua. Mathmatica, 46, 1(2000), 3- 24.

[4] B.C. Dhage, On generalized metric spaces and topological structure, II, Pure and Applied Mathematika Sciences, .40, No.1-2(1994), 37-41.

[5] B.C. Dhage, On continuity of mappings in D-metric spaces, Bulletin of the Calcutta Mathematical Society, 86, No.6 (1994), 503-508.

[6] L.G. Huang and X. Zhang, Cone metric spaces and fixed point theorems of contractive mappings, J. Math. Anal. Appl., 332(2007), 1468-1476.

[7] S. Sedghi, N. Shobe and H. Zhou, A common fixed point theorem in $D^{*}$-metric spaces, Fixed Point Theory and Application, (2007), 1-13.

[8] S. Sedghi, N. Shobe and S. Sedghi, Common fixed point theorems for two mappings in $D^{*}$-metric spaces, JPRM, Vol. 4(2008), 132-142.

[9] Z. Mustafa and B. Sims, Some remarks concerning D-metric spaces, International Conference on Fixed Point Theory and Applications, Yokohama, Yokohama, Japan, 189-198, (2004). 


\section{SANDEEP BHATT}

Department of Mathematics

H. N. B. Garhwal University

SRinagar (GARHWAL) UtTarakHand - 246174

INDIA

E-mail address: bhattsandeep1982@gmail.com

\section{Brian Fisher}

Department of Mathematics

UNIVERSITY OF LEICESTER

LEICESTER LE1 7RH

$\mathrm{UK}$

E-mail address: fbr@leicester.ac.uk

\section{Shruti Chaukiyal}

Department of Mathematics

H.N.B. Garhwal University

SRINAGAR (GARHWAL) UtTARAKHAND - 246174

INDIA

E-mail address: chaukiyalshruti26@gmail.com 